\title{
Aspects familiaux du cancer recto-colique sporadique : acquis épidémiologiques récents pour le clinicien
}

Les aspects familiaux des cancers recto-coliques (CRC) ont amené les cliniciens à distinguer trois situations, les deux premières (polyposes adénomateuses et CRC héréditaires sans polypose) étant associées à un contexte familial démontré alors que dans la troisième, qui est la plus fréquente (CRC sporadiques), le caractère familial n'est que probable $(\mathrm{m} / \mathrm{s}$ $n^{\circ} 2$, vol. 10, p. 228): (1) les CRC sur polypose adénomateuse familiale: ils surviennent dans $100 \%$ des cas en l'absence d'exérèse de celle-ci, mais ne représentent qu'environ $0,2 \%$ des CRC de l'ensemble de la population [1]; (2) les CRC héréditaires sans polypose : ils sont encore dénommés syndrome de Lynch - dont il y a deux variétés selon qu'existe aussi ou non un contexte familial de cancer d'autres organes (par ordre de fréquence décroissante: endomètre, arbre urinaire, estomac, grêle, ovaire, pancréas, voies biliaires, système hématopoïétique, peau et larynx) [2] ; trois critères, dits d'Amsterdam, ont été donnés en 1991 au syndrome de Lynch: atteinte d'au moins trois parents par un CRC histologiquement prouvé, l'un étant parent au premier degré des deux autres; atteinte concernant au moins deux générations; reconnaissance d'au moins un cas de CRC avant l'âge de 50 ans. Le syndrome de Lynch - qui est à distinguer du syndrome récemment décrit d'adénomes rectocoliques sessiles à forme héréditaire [3] - représente $4 \%$ à $6 \%$ de l'ensemble des CRC; (3) le CRC dit sporadique ou commun: jadis considéré comme n'ayant pas de contexte héréditaire, 1158 diversement appréciée depuis une vingtaine d'années, le plus souvent au travers d'études rétrospectives. Plusieurs de celles-ci ont conclu à ce que la notion même d'un seul cas de CRC, histologiquement prouvé, chez un parent au premier degré multipliait le risque personnel de CRC, pour un sujet donné, par un facteur de 1,8 à 8 [4-6]. On conçoit tout l'intérêt d'affirmer la réalité ou non d'un tel risque dans un cancer très fréquent et dont la prévention est assurée par le dépistage des adénomes.

Le travail récent de Fuchs et al. [7] (Université Harvard et Institut du Cancer Dana-Farber, Boston, USA) est, en matière de CRC sporadique, d'une très grande importance épidémiologique et pratique. Ils ont conduit une étude prospective sur deux cohortes de professionnels de santé, l'une de 32085 hommes de 40 à 75 ans, l'autre de 87031 femmes de 30 à 55 ans, qui n'avaient jamais eu d'examen endoscopique de l'intestin et ont été interrogés régulièrement tous les deux ans, à partir de 1982 sur la survenue chez eux-mêmes d'un CRC pendant la période d'étude, et/ou sur l'existence (antérieure ou incidente pendant l'étude) d'un CRC chez leurs parents au premier degré, sur leurs habitudes alimentaires et de vie (graisses animales, fibres alimentaires, viandes rouges, tabac, activité physique), et sur divers facteurs incriminés dans la genèse ou la protection vis-à-vis du CRC tels que la consommation régulière d'aspirine, de calcium et de vitamines antioxydantes. Ont été exclus les sujets ayant un contexte de polypose adé- nomateuse familiale, de rectocolite hémorragique, des antécédents personnels d'adénome rectocolique ou de cancer, notamment de CRC.

Un CRC a été diagnostiqué, au cours de la période d'étude, chez 148 hommes et 315 femmes des deux cohortes: chez ces 463 sujets, la fréquence d'un antécédent de CRC chez au moins un de leurs parents au premier degré a été de $17 \%$, alors qu'elle était significativement plus faible $(9,4 \%$ pour les hommes et $10 \%$ pour les femmes) dans l'ensemble de la cohorte. Le risque relatif de cancer colique ajusté pour l'âge était, chez les sujets ayant au moins un parent au premier degré atteint, de 1,72 (intervalle de confiance-IC - à $95 \%: 1,34$ à 2,19$)$; il était de 2,75 (IC: 1,34 à 5,63) chez ceux ayant au moins deux parents au premier degré atteints. Chez les sujets de moins de 45 ans, ce même risque relatif était de 5,37 (IC: 1,98 à $14,6)$ avec un parent atteint, mais il diminuait régulièrement et significativement avec l'âge, pour revenir à 1 chez les sujets de 65 ans ou plus. Aucun des autres facteurs étudiés, y compris les habitudes alimentaires et de consommation médicamenteuse, n'avait d'influence significative sur le risque de CRC, qu'il y eût ou non un contexte familial. Dans les deux cohortes étudiées, l'augmentation du risque en fonction du contexte familial concernait le cancer colique, mais pas le cancer rectal; cette augmentation était, en revanche, sans relation avec le siège droit ou gauche du cancer colique. Afin d'éliminer le biais par lequel le risque excessif apparent de cancer colique chez les 
sujets à contexte familial de CRC pouvait n'être dû qu'à une surveillance plus étroite liée à ce contexte, une analyse excluant les sujets atteints pouvant n'avoir été détectés que par ce type de dépistage a confirmé que le risque relatif restait significativement élevé à 2 (IC: 1,32 à 3,03).

Ce travail prospectif [7] confirme l'hypothèse d'un risque élevé de cancer colique chez un sujet ayant ou ayant eu au moins un parent au premier degré atteint de CRC. La corrélation, lors du diagnostic, entre les âges du sujet et de son parent n'y a pas été étudiée, mais nous avions montré [5], dans une étude rétrospective, que ces âges n'étaient pas significativement différents et que, partant, le dépistage du CRC - ou mieux sa prévention par le dépistage des adénomes- chez un individu donné devait commencer d'autant plus tôt que son parent au premier degré avait été atteint plus jeune: le travail de Fuchs et al. [7] confirme ce point. Même si les sujets ayant un syndrome de Lynch n'en ont pas été clairement exclus, il est très peu probable que cette réserve ait pu interférer avec leurs résultats [7], puisque seulement sept sujets (tous des femmes) avaient plus de deux parents au premier degré atteints. En pratique, il paraît raisonnable de commencer la prévention et/ou le dépistage du CRC entre 30 et 45 ans, lorsque le parent au ler degré a été lui-même atteint à moins de 50 ans. Chez les sujets de plus de 65 ans, la prévention/dépistage reste indiquée en cas d'antécédents familiaux, même si, dans l'étude de Fuchs et al. [7], l'augmentation, propre à cet âge, du risque de CRC a probablement masqué celle liée au contexte familial.

Un autre travail récent [8], mené parmi des sujets ayant un contexte familial de CRC sans polypose, a, quant à lui, comparé la fréquence des adénomes colo-rectaux chez ceux ayant un syndrome de Lynch défini selon les critères d'Amsterdam, et ceux ayant un contexte familial de type "sporadique" avec atteinte d'un ou deux parents au premier degré ou, à défaut, deux parents de générations différentes. Le risque d'adénome colique était plus élevé (risque relatif $1,76, p<0,02$ ) dans le cadre du syndrome de Lynch (26,8\% d'adénomes) qu'en cas de contexte familial de type sporadique, où la fréquence des adénomes était de 21,3 $\%$. La proportion d'adénomes du côlon droit était plus élevée dans le syndrome de Lynch $(47,1 \%)$ que dans le contexte sporadique $(27,3 \%)$. Les variables indépendantes exposant à un risque accru d'adénome étaient l'âge (prévalence de $9 \%$ à 35 ans, s'élevant à plus de $45 \%$ au-dessus de 55 ans - le risque doublant approximativement à chaque décennie), le sexe (prévalence deux fois plus grande chez l'homme que chez la femme) et le nombre de générations atteintes. Ce travail établit qu'en dehors du syndrome de Lynch, le contexte familial d'adénome recto-colique est une réalité clinique au cours du CRC dit sporadique; l'adénome y est situé en amont de l'angle gauche plus d'une fois sur quatre, ce qui plaide en faveur d'un dépistage par coloscopie totale de principe dans cette population.

La synthèse de ces deux études [7, 8] est d'une grande importance pratique en matière de prévention du CRC "sporadique" $\left(\mathrm{m} / \mathrm{s} n^{\circ} 5\right.$, vol. 8 , p. 496). Elle valide la pratique, en clinique courante, de la coloscopie à visée préventive (dépistage des adénomes), chez les sujets asymptomatiques ayant un contexte de CRC même chez seulement un parent au premier degré; l'âge auquel la première coloscopie devra être pratiquée sera d'autant plus jeune que le parent aura été lui-même atteint plus jeune, en particulier avant 50 ans

\section{RÉFÉRENCES}

1. Thomas, G. Dix ans de recherche sur les prédispositions génétiques au développement des tumeurs. médecine/sciences 1995 . $11: 336-48$

2. Lynch HT, Smyrk TC, Watson P, Lanspa SJ, Lynch JF, Lynch PM, Cavalieri RJ, Boland CR. Genetics, natural history, tumor spectrum, and pathology of hereditary nonpolyposis colorectal cancer: an updated review. Gastroenterology 1993; 104: 1535-49.

3. Lynch HT, Smyrk T, Lanspa SJ, Kriegler M, Lynch JF, Appelman HD. Flat adenomas in a colon cancer-prone kindred. J Natl Cancer Inst 1988; 80: 278-82.

4. Guillem JG, Forde KA, Trat MR, Neugut Al, O'Toole KM, Diamond BE. Colonoscopic screening for neoplasms in asymptomatic first-degree relatives of colon cancer patients: a controlled prospective study. Dis Colon Rectum 1992; 35 : 523-9.

5. Maire $P$, Morichau-Beauchant M, Drucker J, Barboteau MA, Barbier J, Matuchansky C. Prévalence familiale du cancer du côlon et du rectum: résultats d'une enquếte "cas-témoins" de 3 ans. Gastroenterol Clin Biol 1984; 8: 22-7.

6. Slattery ML, Kerber RA. Family history of cancer and cancer colon risk: the Utah population database. J Natl Cancer Inst 1994; 86: 1618-26.

7. Fuchs CS, Giovannucci EL, Colditz GA, Hunter DJ, Speizer FE, Willett WC. A prospective study of family history and the risk of colorectal cancer. $N$ Engl J Med 1994; 331: 1669-74.

8. Gaglia P, Atkin WS, Whitelaw S, Talbot IC, Williams CB, Northover JMA, Hodgson SV. Variables associated with the risk of colorectal adenomas in asymptomatic patients with a family history of colorectal cancer. Gut 1995; 36 : 385-90.

\section{Claude Matuchansky}

Professeur des universités, praticien hospitalier, service de gastroentérologie et Inserm U. 290, hôpital St-Lazare, 107 bis, rue du faubourg Saint-Denis, 75475 Paris Cedex, France. 